\title{
Die Rolle des Internets in deutschen Wahlkämpfen
}

\author{
Andreas Jungherr
}

\begin{abstract}
Kernaussagen
Um die Potentiale des Internets für Wahlkämpfer in Deutschland $\mathrm{zu}$ verstehen, ist es notwendig, differenziert über die Funktionen des Internets für politische Kampagnen nachzudenken. Hierbei bietet sich die Analyse von drei Funktionsweisen an: 1. Internetdienste als Werkzeuge, um die Sichtbarkeit politischer Akteure und ihrer Inhalte im Informationsraum Internet zu gewährleisten; 2. Die Unterstützung der Infrastruktur einer politischen Kampagne durch digitale Werkzeuge und das Internet; 3. Die symbolische Sichtbarmachung von politischer Unterstützung und des Momentums von Kampagnen und die damit verbundene Möglichkeit, darauf folgender Berichterstattung traditioneller Medien. Die Potenziale dieser Funktionsweisen sind jeweils abhängig von den spezifischen politischen, rechtlichen, kulturellen und historischen Kontexten in denen Kampagnen und Kandidaten agieren. Sie können sich je nach Kandidat, Partei oder Land unterscheiden. In dem vorliegenden Artikel werden diese Funktionen des Internets für politische Kampagnen an den Beispielen der Präsidentschaftskampagnen Barack Obamas und deutscher Wahlkämpfe diskutiert.
\end{abstract}

\section{Das Wahlkampfinstrument Internet}

Das Internet ist spätestens seit der viel kommentierten Kampagne Barack Obamas zur US-Präsidentschaftswahl 2008 aus internationalen Wahlkämpfen nicht mehr wegzudenken. Trotz der weit verbreiteten Nutzung des Internets in Wahlkämpfen gibt es aber bis heute keine Beispiele in denen es politischen Akteuren gelang, das Internet mit ähnlichem Erfolg wie Barack Obama zu nutzen. Dies gilt auch für den Einsatz des Internets in deutschen Wahlkämpfen.

Kommunikationsberater und Kommentatoren in den Medien führen die relativ enttäuschenden Online-Anstrengungen von deutschen Kandidaten und Parteien in der Regel darauf zurück, dass die verantwortlichen politischen Akteure das Medium Internet nicht verstünden und so wesentliche Potentiale des Internets für ihre Kampagnen verschenkten. Diese Argumentation basiert auf einer einfachen Annahme: die erfolgreiche Nutzung eines Onlinewerkzeugs in einem spezifischen politischen, rechtlichen, kulturellem und historischem Kontext lasse sich kontextunabhängig reproduzieren. Barack Obama nutzte das Internet erfolgreich in seiner Kampagne also müssen sich seine Erfolge auch von anderen Kandidaten in anderen Kontexten reproduzieren lassen. Bleibt in diesen Fällen der Erfolg aus so lässt sich dies auf die fehlerhafte Nutzung des Internets durch diese Kandidaten zurückführen.
Unbeachtet hierbei bleibt die Frage, ob es für Wahlkampagnen in den USA bestimmte Kontextbedingungen gibt, die dort wesentlich zum Bedeutungsgewinn des Internets in Wahlkämpfen beitrugen. Kontextbedingungen, die nicht notwendigerweise in anderen Ländern gegeben sind. Diese Position lässt sich als Amerikanisierung 2.0 bezeichnen.

Diese kontextunabhängige Betrachtung bringt offensichtliche Defizite mit sich. Alternativ bietet die Diskussion unterschiedlicher Onlinekampagnen anhand von drei grundlegenden Funktionen des Internets für Wahlkämpfe mehr Potential (Jungherr, 2012; Jungherr \& Schoen, 2013).

Wahlkämpfe in den USA zeigten drei Funktionen, die das Internet in Kampagnen erfüllen kann:

1. Sichtbarkeit des politischen Akteurs und seiner Inhalte im Informationsraum Internet;

2. Die Unterstützung der Infrastruktur einer politischen Kampagne durch digitale Werkzeuge und das Internet;

3. Die symbolische Sichtbarmachung von politischer Unterstützung und des Momentums von Kampagnen und die damit verbundene Möglichkeit, dass traditionelle Medien darüber berichten.

Dieser Artikel stellt diese drei Funktionen vor und illustriert sie exemplarisch mit Beispielen aus US-Wahlkämpfen. In der Folge werden diese Funktionen für deutsche Wahlkämpfe diskutiert.

\section{Sichtbarkeit im Informationsraum}

Das Internet entwickelt sich für Menschen zu einer immer bedeutenderen Informationsquelle. Auch für politische Akteure sind ihre Sichtbarkeit und die Sichtbarkeit ihrer Botschaften im Informationsraum Internet von wachsender Bedeutung. Für Parteien und Kandidaten bieten ihre Webseiten, E-Mail-Verteiler und Angebote auf anderen Onlinediensten einfache Wege, um ihre Inhalte, Positionen und Kampagnenbotschaften für Unterstützer oder interessierte Internetnutzer aufzuarbeiten und zugänglich zu machen. Voraussetzung für die erfolgreiche Nutzung dieser Funktion ist jedoch die bewusste Gestaltung politischer Onlineangebote, so dass diese tatsächlich im Internet sichtbar sind.

Politische Akteure haben unterschiedliche Möglichkeiten, ihre Angebote im Internet zu positionieren. Einerseits können sie eigene Angebote redaktionell betreiben (z.B. Webseiten, EMail-Verteiler, Profile auf unterschiedlichen Sozialen Netzwerkplattformen). Andererseits können sie verschiedene Wege nutzen, um auf ihre Angebote aufmerksam zu machen (z.B. Anzeigen auf Suchmaschinen oder Sozialen Netzwerkplattformen, die Ermutigung zur Verlinkung ihrer redaktionellen Angebote auf Blogs oder Sozialen Netzwerkprofilen durch 
Dritte). Hierbei können politische Akteure die Sichtbarkeit ihrer Onlineangebote aktiv und ohne großen Aufwand fördern. Dies kann sowohl durch die bewusste redaktionelle und kanalspezifische Betreuung ihrer Onlinegebote erfolgen als auch durch die technische Gestaltung der Angebote. Die Suchmaschinenoptimierung politischer Angebote soll gewährleisten, dass die entsprechenden Angebote von Internetsuchmaschinen zu, für den politischen Akteur, relevanten Suchbegriffen (z.B. „Bundestagswahl“, „Wahlkampf“, „Energiewende“) prominent gelistet werden.

In der Regel sind mit vier unterschiedlichen Nutzergruppen der Angebote politischer Akteure zu rechnen: politische Unterstützer, zufällige Besucher, die über die Suche nach einem politischen Begriff auf das Onlineangebot gestoßen sind, politische Mitbewerber und Journalisten (Bimber \& Davis, 2003). Die unterschiedlichen Bedürfnisse dieser Nutzergruppen sollte die Gestaltung, redaktionelle Betreuung und die Bewerbung der Onlineangebote bestimmen.

Trotz des offensichtlichen Potentials, durch einige gezielte Maßnahmen die Sichtbarkeit eigener redaktioneller Inhalte im Internet positiv zu beeinflussen, sind politische Akteure in Deutschland in diesem Bereich noch sehr zurückhaltend. Webseiten, die Nutzung von E-Mail-Verteilern und Profilen auf Sozialen Netzwerkplattformen sind zwar inzwischen weit verbreitet, die aktive Nutzung dieser Werkzeuge, um im Internet nicht nur zu der direkten Suche nach den Namen von Politikern oder Parteien zu erscheinen ist jedoch noch nicht selbstverständlich. Zur Zeit ist die Präsenz und der Einfluss von politischen Akteuren im Informationsraum Internet in Deutschland noch deutlich untererforscht. Bisherige Ergebnisse deuten jedoch darauf hin, dass politische Akteure die hier bestehenden Möglichkeiten nur ungenügend nutzen, um ihre Inhalte und Internetangebote prominent $\mathrm{zu}$ positionieren (Jungherr, Schoen \& Ludwigs, 2011).

Eine mögliche Erklärung hierfür ist die im internationalen Vergleich relativ geringe Nutzung des Internets als Quelle politischer Informationen in Deutschland. Die Zahl der Deutschen, die angeben, das Internet zu nutzen, um sich über den Verlauf der Kampagne zu informieren bewegte sich von 11\% im Jahr 2002 auf 19\% im Jahr 2009 (Faas \& Partheymüller, 2011; Huber, 2009) während im gleichen Zeitraum die Zahl der aktiven Internetnutzer von $44 \%$ auf $67 \%$ stieg (van Eimeren $\&$ Frees, 2011). Die relativ niedrige politische Internetnutzung in Deutschland kann also nicht einer niedrigen Verbreitung des Internets zugeschrieben werden. Zum Vergleich, in den USA nutzten 2010 kurz vor den Kongresswahlen 58\% Prozent der Bevölkerung das Internet, um sich politisch zu informieren (Smith, 2011). Die Zurückhaltung von politischen Akteuren, in ihre Sichtbarkeit im Informationsraum Internet zu investieren könnte also nicht nur ihrem Desinteresse gegenüber dem neuen Kommunikationsumfeld geschuldet sein, sondern unter Umständen auch ihrer Einschätzung, dort nur begrenzt Menschen erreichen zu können.

\section{Unterstützung der Infrastruktur einer Kampagne}

Digitale Werkzeuge und Onlinedienste werden zunehmend erfolgreich von politischen Kampagnen in den USA genutzt, um Elemente der klassischen Kampagnenführung zu unterstützen und effizienter zu gestalten. Dazu gehört das Fundraising über personalisierte E-Mails, die Verbesserung der Auswahl potentieller Wähler, die daraufhin von der Kampagne kontaktiert werden oder das Testen unterschiedlicher Anspracheformen für Unterstützer und potentielle Wähler. Matthew Hindman hat diese Form der politischen Internetnutzung als Unterstützung und Transformation der Infrastruktur politischer Kampagnen bezeichnet (Hindman, 2009). Diese Funktion des Internets wird zumindest in der deutschsprachigen Diskussion über die Rolle des Internets für politische Kampagnen weitgehend ignoriert. Dennoch ist diese Funktion, zumindest in den USA, wesentlich für die gestiegene Bedeutung des Internets in politischen Kampagnen verantwortlich.

Die Kampagnen Barack Obamas 2008 und 2012 nutzten das Internet gezielt, um Fundraising, Wähler-Targeting und die Koordination von Kampagnenunterstützern zu verbessern. Der deutliche Beitrag, den das Internet in diesen Bereichen lieferte, führte dazu, dass die Obama-Kampagne den Leiter des Internetteams auf der höchsten Leitungsebene der Kampagne einband, gleichberechtigt zu den Leitern traditioneller Kampagnenelemente. Diese prominente Positionierung führte zu dem gesteigerten Einfluss der Onliner auf die gesamte Kampagnenstrategie (Kreiss, 2012). In Deutschland gibt es keine vergleichbar prominente Positionierung der Onlineteams politischer Kampagnen. In der Regel sind die Internet-Verantwortlichen der Parteien auf Referentenebene in den Kommunikationsabteilungen von Parteien und Kampagnen angesiedelt. Ihr Einfluss auf der Leitungsebene der Kampagne ist also in der Regel eher gering. Dies erschwert die Koordination klassischer Kampagnenelemente mit Online-Elementen.

In den USA zeigten sich die Fundraising-Potentiale des Internets für politische Kampagnen bereits seit Anfang der zweitausender Jahre. Frühe Erfolge der Kampagne Howard Deans im amerikanischen Präsidentschaftsvorwahlkampf 2002/3 wurden von den Kampagenen Barack Obamas 2008 und 2012 als Beispiel genommen. Zusätzlich testete die Obama-Kampagne akribisch wie digitale Werkzeuge zu einer Erhöhung der Spenden ihrer Unterstützer führen konnten. Entscheidend hierbei waren Kleinspenden, die prominent auf der Webseite und über den E-Mail Verteiler der Obama-Kampagne eingeworben wurden. Hierbei testete die Kampagne in Experimenten gezielt unterschiedliche Anspracheformen (z.B. Platzierung des Spenden-Buttons auf der Webseite oder unterschiedliche Betreffzeilen, Absender und Formulierung von EMails, die um Spenden baten) auf ihr Potential, Spenden bei unterschiedlichen Unterstützergruppen zu generieren. Der Erfolg dieses quasi wissenschaftlichen Vorgehens machte die Internet-Abteilung der Obama-Kampagne zu einem entscheidenden Ressourcenfaktor in der Gesamtkampagne und verstärkte dadurch ihren Einfluss (Issenberg, 2012 b; Kreiss, 2012; Madrigal, 2012). In Deutschland spielt das Internet für 
keine Partei bisher eine vergleichbare Rolle in der Sammlung von Spenden was es die Internet-Zuständigen der Kampagnen deutlich schwerer macht, ihre Bedeutung für die Gesamtkampagne zu quantifizieren.

Ein weiterer entscheidender Beitrag, den digitale Werkzeuge zu US-amerikanischen Kampagne liefern ist ihre Unterstützung in der Sammlung, Verarbeitung und Nutzbarmachung von Daten über potentielle Wähler. In den letzten Jahren wurde für politische Kampagnen in den USA der persönliche Wählerkontakt mit Freiwilligen, sei es im Tür-zu-TürWahlkampf oder durch Telefonanrufe immer wichtiger. Da diese Kontakte kostenintensiver sind als traditionelle Postsendungen oder Robocalls wird es für Kampagnen immer wichtiger die Kontaktversuche auf Ansprechpartner zu konzentrieren, die eine hohe Wahrscheinlichkeit haben, tatsächlich zur Wahl zu gehen und dann auch den richtigen Kandidaten zu wählen. Seit 2005 investierte die demokratische Partei bewusst in den Aufbau einer zentralen Datenbank, die Kandidaten und Kampagnen der Demokraten auf unterschiedlichen politischen Ebenen zur Lizensierung zur Verfügung steht. Diese Datenbank war eine der Grundlagen für Barack Obamas erfolgreicher Tür-zu-Tür-Mobilisierung in seinen Kampagnen 2008 und 2012 (Issenberg, 2012 a; Kreiss, 2012; Nielsen, 2012). Auch hier bietet das Internet und digitale Werkzeuge amerikanischen Kampagnen eine klar bezifferbare Unterstützung in Kernbereichen politischer Kampagnenführung. Allerdings handelt es sich auch hier um ein Kampagnenelement, das in Deutschland nur bedingt Anwendung findet. Gründe hierfür liegen einerseits im Datenschutzrecht, dass einen großen Teil der in den USA von Kampagnen über potentielle Wähler gesammelten Daten in Deutschland für Kampagnen nicht verfügbar macht und andererseits in der Kampagnenkultur in Deutschland, die bisher nicht auf großflächig koordinierten Tür-zu-Tür-Wahlkämpfe setzt. Eine Neuerung hierzu könnte die SPD-Kampagne zur Bundestagswahl 2013 bieten, in der die Partei bewusst verstärkt auf den durch das Internet unterstützten und koordinierten Tür-zu-Tür-Wahlkampf setzt (Kroke, 2013; Richel, 2013).

Die klar bezifferbare Unterstützung der Infrastruktur politischer Kampagnen in den USA hat das Internet dort zu einem Kernelement politischer Kampagnen gemacht. Solange in Deutschland Kandidaten oder Parteien nicht Ansätze entwickeln mit denen sie Kernfunktionen ihrer Kampagnen entscheidend durch digitale Werkzeuge verbessern können wird es Internet-Verantwortlichen schwer fallen, in der Führungsebene von Kampagnen als gleichberechtigte Teilnehmer akzeptiert zu werden. Erschwert wird dies dadurch, dass die Bereiche in denen das Internet in den USA die größten Erfolge für Kampagnen brachte in Deutschland für Kampagnen aus datenschutz-, parteienfinanzierungsrechtlichen und kulturellen Gründen deutlich geringere Bedeutung haben als in den USA. Deutsche Kampagnen müssen hier also neu denken und können nicht einfach nur bestehende Lösungen aus den USA importieren.

\section{Symbol für Unterstützung und das Momentum von Kampagnen}

Eine dritte Funktion des Internets für politische Kampagnen ist die symbolische Sichtbarmachung politischer Unterstützung und des Momentums einer Kampagne. Die meisten Angebote politischer Kampagnen im Internet sind öffentlich leicht zählbar. Seien es die Unterstützer auf Facebook oder seien es die Klicks auf einem YouTube Video. Diese leichte Zählbarkeit bietet sich für die journalistische Berichterstattung über die Erfolge von Kampagnen und Kandidaten online an. Dies führt in den Medien zunehmend zu einer Berichterstattung über das digitale horse-race, also eine Berichterstattung, die sich auf die relative Entwicklung der Unterstützerzahlen oder Interaktionszahlen auf den Profilen von Kandidaten oder Parteien konzentriert. Am ehesten ist dies mit der oft kritisierten klassischen horse-race Berichterstattung über relative Veränderungen in Umfrageergebnissen zu vergleichen. Die Funktion der Unterstützerzahlen auf Sozialen Netzwerkplattform für Kampagnen liegt also in ihrer Spiegelung in Presseberichterstattung über diese Zahlen und nicht in der direkten Mobilisierung von Wählern oder Unterstützern.

Politiker nutzen das Internet und ihr persönliches Nutzungsverhalten zunehmend auch als Element ihres Kandidatenprofils. Sie beschränken sich nicht nur darauf einen bestimmten Onlinedienst (z.B. Twitter) zu nutzen sondern erzeugen aktiv Medienberichte, sei es als Namensbeitrag (Altmaier, 2011) oder als Interview (Diehl \& Nelles, 2012), in denen sie auf diesem etablierten Kommunikationskanal über die Bedeutung des Internets für ihr persönliches Kommunikationsverhalten und ihr Politikverständnis sprechen. Auch hier dient die Nutzung des Internets der Erzeugung positiver Medienberichterstattung und weniger der direkten Wählermobilisierung.

Zunehmend gelingt es auch politischen Aktivisten Onlineartefakte zu schaffen, die gesellschaftliche Relevanz ihrer Anliegen visualisieren und daraufhin von traditionellen Medien als Anlass für Berichterstattung genommen werden. Seien es Video Clips von Demonstrationen (Jungherr, 2012 b) oder erfolgreiche Online-Petitionen (Jungherr \& Jürgens, 2011). Auch in diesem Fall ist die Funktion des Internets in erster Linie eine symbolische, die daraufhin in der Berichterstattung von traditionellen Massenmedien allgemeine Verbreitung erfährt.

Dies ist bisher die in Deutschland am ehesten wahrnehmbare Funktion des Internets für politische Kampagnen. Sollte sich die Funktion des Internets für politische Kampagnen in Deutschland allerdings bis auf weiteres nur auf die Sichtbarmachung politischer Unterstützung beschränken, dann ist zu erwarte, dass das Internet bloß Beiwerk politischer Kampagnen in Deutschland bleibt und nicht kampagnenmitbestimmendes Kernelement von Wahlkämpfen wird. 


\section{Amerikanisierung 2.0?}

Im Vergleich zu den USA erfüllt das Internet in deutschen Kampagnen also eher nur eine beschränkte Funktion. Dies erklärt sich jedoch nicht notwendig dadurch, dass Parteien und Kandidaten in Deutschland die Potentiale des Internets nicht verstünden. Es liegt eher daran, dass Kampagnen in Deutschland unter anderen, dem Internet weniger freundlichen, Kontextbedingungen agieren als Kampagnen in den USA.

Zum einen verfügen Kampagnen in den USA über weitaus größere Budgets als in Deutschland. So konnte Barack Obama 2008 über 500 Millionen Dollar in Spenden sammeln während die größten Parteien in Deutschland im Bundestagswahlkampf 2009 mit 29 (SPD) bzw. 26,5 (CDU/CSU) Millionen Euro ihren Wahlkampf bestritten (Johnson, 2011; Tenscher, 2011). In den USA ist es Kampagnen also durchaus möglich relevante Beträge in die Entwicklung und Verfeinerung digitaler Werkzeuge zu investieren, während dies in Deutschland oft schon am Kampagnenbudget scheitert. Zusätzlich werden in den USA Präsidentschaftskampagnen über einen deutlichen längeren Zeitraum geführt als deutsche Bundestagswahlkämpfe deren heiße Mobilisierungsphasen sich auf wenige Wochen konzentriert. In den USA haben Kampagnenmacher also mehr Zeit, Beziehungen zu Unterstützern aufzubauen und sie mit langfristigen Spendenstrategien an sich zu binden. Wie oben bereits angesprochen, sind die Parteien in Deutschland auf Grund der politischen Kultur und des Datenschutzrecht nicht in der Lage, ähnlich detaillierte Datensätze über potentielle Wähler anzulegen wie Parteien in den USA. Auch hier bietet das Internet also keine entscheidende Kampagnenunterstützung. Außerdem ist das Internet ein Medium, das Motivation auf Seiten des Nutzers voraussetzt. In Deutschland werden Kampagnen allerdings seit einigen Jahren überwiegend deeskalierend, oder asymmetrisch demobilisierend, geführt. Dies führt unter Umständen zu der im internationalen Vergleich geringen Zahl von Deutschen, die das Internet nutzt, um sich über den Verlauf der Kampagne zu informieren. Auch dies ist ein entscheidender Unterschied zu Kampagnen in den USA, die zunehmend in einem symbolisch stark polarisierten Klima stattfinden.

Dies zeigt, dass das Internet deutschen Kampagnen, zumindest zur Zeit, in dem politischen, rechtlichen und kulturellen Kontext deutscher Wahlkämpfe andere Potentiale bietet als Kampagnen in den USA. Die relativ beschränkte Nutzung des Internets in deutschen Kampagnen ist also weder überraschend, noch liegt sie notwendiger Weise am mangelnden Verständnis der Parteien oder Kampagnen sondern liegt vielmehr an dem unterschiedlichen Potential des Internets für politische Kampagnen abhängig von den entsprechenden politischen, rechtlichen und kulturellen Kontexten. Das heißt jedoch nicht, dass es in Zukunft nicht auch Kampagnen in Deutschland gelingen kann das Internet verstärkt in die Kampagnenführung einzubinden. Allerdings wird dies wohl andere Gestalt annehmen als in den USA.

\section{Das Internet in deutschen Wahlkämpfen}

Bei der Interpretation der Rolle des Internets in politischen Kampagnen ist es nicht sinnvoll, die in anderen Wahlkämpfen gezeigten Potentiale von Onlinetools als allgemein gültigen Standard zu definieren und andere Akteure, die diese Potentiale nicht ausschöpfen als unverständig zu bezeichnen. Vielmehr geht es darum, die Potentiale des Internets für Kampagnen in unterschiedlichen politischen, rechtlichen und kulturellen Kontexten realistisch einzuschätzen und vor diesem Hintergrund zu diskutieren. Das Internet hat unterschiedliche Potentiale für unterschiedliche politische Akteure in unterschiedlichen Kontexten und zu unterschiedlichen Zeitpunkten.

In diesem Artikel wurden drei Funktionen aufgezeigt, die dass Internet in Wahlkämpfen in den USA erfüllt. Diese waren:

1. Sichtbarkeit des politischen Akteurs und seiner Inhalte im Informationsraum Internet;

2. Die Unterstützung der Infrastruktur einer politischen Kampagne durch digitale Werkzeuge und das Internet;

3. Die symbolische Sichtbarmachung von politischer Unterstützung und des Momentums von Kampagnen und die damit verbundene Möglichkeit, dass traditionelle Medien darüber berichten.

Der Artikel zeigte, dass das Internet in deutschen Wahlkämpfen nur die dritte Funktion mit nennenswertem Erfolg erfüllt. Wie der Artikel aber auch zeigte liegt dies wohl weniger an dem mangelnden Verständnis des Internets durch deutsche Wahlkämpfer sondern an den spezifischen Kontextbedingungen unter denen in Deutschland Wahlkampf gemacht wird. Die Wahlkämpfe des Jahres 2013 werden zeigen, ob dieses Muster auch dieses Jahr hält oder ob es den Wahlkämpfern gelingt, neue Potentiale des Internets in deutschen Kampagnen zu erschließen.

\section{Literatur:}

Altmaier, P. (2011, Oktober 13). Mein neues Leben unter Piraten. Frankfurter Allgemeine Zeitung. Retrieved from www.faz.net/aktuell/feuilleton/debatten/digitales-denken/politik-und-internet-mein-neues-lebenunter-piraten-11493287.html.

Bimber, B., \& Davis, R. (2003). Campaigning online: the Internet in U.S. elections. Oxford: Oxford University Press.

Diehl, J., \& Nelles, R. (2012, März 28). Hannelore Kraft im Interview: "Ich will einen Live-Ticker meines Lebens." Spiegel Online. Retrieved from http://www.spiegel.de/politik/deutschland/interview-mit-nrwministerpraesidentin-hannelore-kraft-spd-a-824125.html.

Faas, T., \& Partheymüller, J. (2011). Aber jetzt?! Politische Internetnutzung in den Bundestagswahlkämpfen 2005 und 2009. In E.-J. Schweitzer \& S. Albrecht (Hrsg.), Das Internet im Wablkampf: Analysen zur Bundestagswahl 2009 (S. 119-135). Wiesbaden: VS Verlag für Sozialwissenschaft.

Hindman, M. (2009). The myth of of digital democracy. Princeton, NJ: Princeton University Press.

Huber, S. (2009). Das Internet als Erweiterung des politischen Kommunikationsraums: Nutzerakzeptanz und Nutzungsprofile und ibre Folgen für die politische Partizipation. Unveröffentlichte Dissertation, OttoFriedrich-Universität, Bamberg.

Issenberg, S. (2012 a, Oktober 29). Obama does It better. Slate.

Retrieved from http://www.slate.com/articles/news_and_politics/vic- 
tory_lab/2012/10/obama_s_secret_weapon_democrats_have_a_massive_advantage_in_targeting_and.html.

Issenberg, S. (2012 b). The victory lab: the secret science of winning campaigns. New York, NY: Crown Publishing Group.

Johnson, D. W. (2011). Campaigning in the twenty-first century: a whole new ballgame. New York, NY: Routledge.

Jungherr, A. (2012 a). Online campaigning in Germany: The CDU online campaign for the general election 2009 in Germany. German Politics 21(3), 317-340. doi: 10.1080/09644008.2012.716043.

Jungherr, A. (2012 b). The German federal election of 2009: the challenge of participatory cultures in political campaigns. Transformative Works and Cultures, (10). doi: 10.3983/twc.2012.0310.

Jungherr, A. \& Schoen H. (2013). Das Internet in Wablkämpfen: Konzepte, Wirkungen und Kampagnenfunktionen. Wiesbaden: Springer VS.

Jungherr, A., Schoen, H. \& Ludwigs, D. J. (2011). Sichtbarkeitsreport: Wie sichtbar sind die Webseiten von politischen Parteien für Suchmaschinen? Retrieved from http://sichtbarkeitsreport.de/.

Jungherr, A. \& Jürgens, P. (2011). E-Petitionen in Deutschland: Zwischen niedrigschwelligem Partizipationsangebot und quasi-plebiszitärer Nutzung." Zeitschrift für Parlamentsfragen (3), 521-534.

Kreiss, D. (2012). Taking our country back: the crafting of networked politics from Howard Dean to Barack Obama. Oxford: Oxford University Press.

Kroke, C. (2013, März 22). Politik auf Augenhöhe. spd.de. Retrieved from http://www.spd.de/94042/20130322_kampa_2013.html.

Madrigal, A. C. (2012, November 16). When the nerds go marching in. The Atlantic. Retrieved from http://www.theatlantic.com/technology/ archive/2012/11/when-the-nerds-go-marching-in/265325/.

Nielsen, R. K. (2012). Ground wars: personalized communication in political campaigns. Princeton, NJ: Princeton University Press.
Richel, M. (2013, April 14). This is the beginning. Retrieved from http:// blog.mathias-richel.de/2013/04/14/this-is-the-beginning/.

Smith, A. (2011). The Internet and campaign 2010. Pew Internet \& American Life Project. Retrieved from http://pewinternet.org/Reports/2011/ The-Internet-and-Campaign-2010.aspx.

Tenscher, J. (2011). Defizitär - und trotzdem professionell? Die Parteienkampagnen im Vergleich. In J. Tenscher (Hrsg.), Superwahljahr 2009: Vergleichende Analysen aus Anlass der Wablen zum Deutschen Bundestag und zum Europäischen Parlamentent (S. 65-95). Wiesbaden: VS Verlag für Sozialwissenschaft.

van Eimeren, B. \& Frees, B. (2011). Drei von vier Deutschen im Netz ein Ende des digitalen Grabens in Sicht? Media Perspektiven (7-8), 334349.

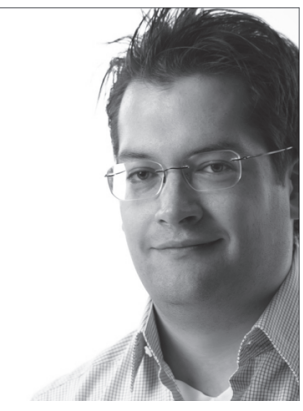

Andreas Jungherr ist Wissenschaftlicher Mitarbeiter am Lehrstuhl für Politische Soziologie an der Universität Bamberg. Dort forscht er über die Rolle des Internets in der politischen Kommunikation und in Wahlkämpfen. Zusammen mit Harald Schoen ist er Autor des Buches Das Internet in Wahlkämpfen: Konzepte, Wirkungen und Kampagnenfunktionen (2013). E-Mail: andreas.jungherr@gmail.com

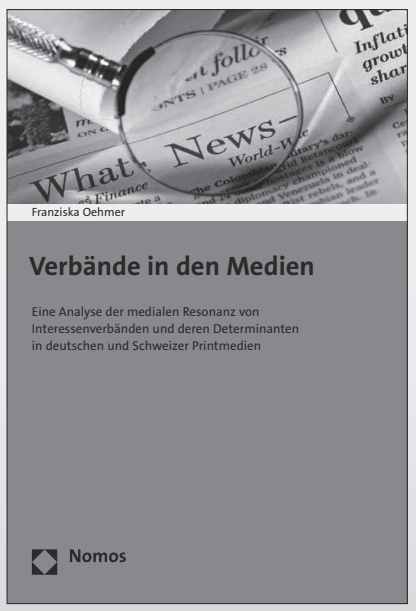

\section{Medienpräsenz von Verbänden}

\author{
Verbände in den Medien \\ Eine Analyse der medialen Resonanz von Interessenverbänden und \\ deren Determinanten in deutschen und Schweizer Printmedien \\ Von Franziska Oehmer \\ 2013, 203 S., brosch., 34,- €, ISBN 978-3-8487-0494-1
}

Gesellschaftliche Wandlungsprozesse lassen die Vermittlungsleistung der Medien zunehmend auch für Interessenverbände an Relevanz gewinnen. Die begrenzten Kapazitäten der Medien ermöglichen es jedoch nicht allen Akteuren präsent zu sein. Die Arbeit identifiziert die Selektionsmechanismen bei der Auswahl von Verbänden in deutschen und Schweizer Tageszeitungen. 